\title{
«Le vieux monde s'écroule, les temps se renouvellent, et une autre vie fleurit» dans le domaine de la santé
}

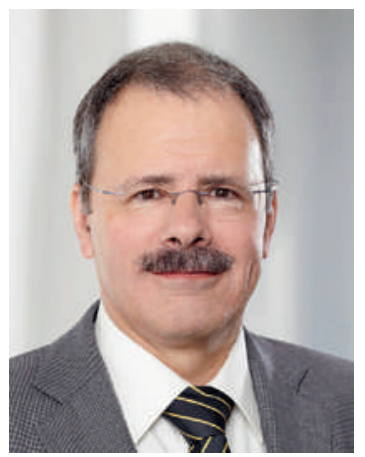

Début juillet, plusieurs sociétés médicales germanophones venant d'Allemagne, d'Autriche, du Tyrol du Sud et du Luxembourg ont retrouvé leurs hôtes suisses à Brunnen, au bord du lac des Quatre-Cantons, pour échanger sur les défis communs des systèmes de santé lors d'une $60^{\text {e }}$ rencontre consultative. Pourquoi cette interprétation - libre - de Guillaume Tell me semble-t-elle juste pour cette conférence?

Quelques tendances très similaires entre les systèmes de santé des cinq pays se sont nettement dégagées à la lecture des thèmes proposés pour la rencontre. En pole position des défis les plus urgents, on trouve la pénurie de jeunes médecins, de plus en plus palpable comme lors de la remise de cabinets ou de postes vacants, et en partie par la sous-couverture médicale de certaines régions. Les jeunes médecins ne sont pas en nombre suffisant pour remplacer les confrères qui cessent leur activité ni pour répondre aux exigences que posent les soins médicaux d'aujourd'hui, notamment en termes de proximité du lieu d'habitation, de disponibilité et de besoins.

\section{«Si nous voulons que tout reste tel quel, il faut que tout change.» (Johann Wolfgang von Goethe)}

A Brunnen, la discussion a abordé tous les aspects du problème. Dans le passé, l'évolution - notamment démographique - de la demande n'a pas été suffisamment prise en compte dans la planification des places de formation. On a pensé à tort que la nouvelle génération de médecins se prêterait aux mêmes conditions extrêmes de travail que ses prédécesseurs. Heureusement, le législateur mais aussi les jeunes médecins font aujourd'hui une lecture différente des limites de ce qui est tolérable. Par ailleurs, aucune mesure n'a été prise pour s'ajuster à la hausse des temps partiels.

Si l'augmentation du nombre de places de formation est sans aucun doute indispensable pour pallier la pénurie, elle ne représente cependant qu'une partie de la solution. Former davantage de médecins, mais également les garder en activité, impliquera aussi des modifications structurelles. Il faudra non seulement proposer un service convenable de garde d'enfants ou instaurer de meilleures conditions cadres pour les services d'urgence et de garde. Mais il faudra aussi que les tendances telles qu'allier flexibilité et mobilité ou pouvoir travailler dans des équipes avec moins de hiérarchie verticale puissent s'intégrer dans les nouvelles structures proposées. Si aujourd'hui les jeunes collègues ne sont plus attirés par la reprise d'un cabinet ou s'ils résistent à s'installer en périphérie, alors ce sont les structures qui doivent changer pour alléger le quotidien.

En arrière-fond de la pénurie de médecins et des discussions itératives sur les coûts, le thème des structures de soins soulève aussi des questions difficiles: faut-il vraiment conserver tous les cabinets? Toutes les offres de soins doivent-elles être disponibles à tout moment et partout? A une époque où les petites épiceries disparaissent parce que les gens n'hésitent pas à parcourir plusieurs kilomètres, ne faudrait-il pas envisager aussi un certain trajet pour une consultation médicale?

Les quatre autres sujets traités lors de la rencontre étaient en partie liés au premier. Mais tant le potentiel des nouvelles technologies qu'une meilleure utilisation des ressources grâce à davantage d'interprofessionnalité n'ont pas été considérés comme des réponses pertinentes à la pénurie de médecins. La notion de «profession libérale» a été reprise à la lumière des tendances actuelles, afin de mieux l'appréhender et la transmettre. Quant à l'assistance au suicide, elle a suscité un débat sur les fondamentaux de notre profession, notamment par ses aspects éthiques et juridiques mais aussi déontologiques.

Tous les sujets évoqués mettent en évidence les nouvelles attentes des jeunes médecins mais aussi celles d'autres groupes professionnels et des patients. Sur certains points, nous devrions procéder à des changements profonds, sur d'autres à des adaptations sans toucher au noyau essentiel. Ces réflexions sur les défis communs nous aident à anticiper les progrès, à oser la rupture et le renouveau, et à avancer dans les rénovations. Nous ne voulons pas laisser des «ruines» à la nouvelle génération de médecins mais faire éclore ensemble une couverture des soins moderne.

Dr Jürg Schlup, président de la FMH

1 «Le vieux monde s'écroule, les temps se renouvellent, et une autre vie fleurit sur les ruines.» (Schiller: Guillaume Tell) 